\title{
An Empirical Research Testing the Effects of Multimodality on English Listening Teaching
}

\author{
Hu Yongjin \\ (School of Foreign Studies, Suzhou University, Suzhou, Anhui, 234000) \\ hyj_dily@163.com
}

\begin{abstract}
The research aims at investigating the effects of different modalities and combinations of them on English Listening Teaching, exploring the factors influencing the pattern of multimodality with optimal function and setting practical example to improve the quality of English Listening Teaching. The result shows that reasonable utilization of the combination of multimodalities can improve learners' listening competence; the choice of the combination of multimodalities depends on the context of situation; visual information can give play to positive function providing that it is complementary to auditory information; In addition, L2 subtitling is more beneficial than $\mathrm{L} 1$ because it causes less lexical interference.
\end{abstract}

Key Words- multimodality; English Listening Teaching; effects; empirical research

\section{INTRODUCTION}

According to modern learning theory of Constructivism, knowledge is acquired by the interaction between subjectivity and objectivity instead of simple transmission between them. Thus, it can only be constructed by learners themselves( Jonassen \& Land, 2000). Moreover, the teaching theory of Constructivism proposes that the function of education is to provide students with fruitful activities rather than mechanically inculcate them knowledge. Students can construct knowledge in the process of interaction and problem-solving( Chen Qi \& Zhang Jianwei, 2003) ${ }^{[1-3]}$. The new teaching method of English Listening based on Multimodal Discourse Analysis Theory is in line with this position and in this study, the effects of different modalities and different combinations of them on English Listening Teaching will be tested ${ }^{[4-6]}$.

\section{STUDIES ON ENGLISH LISTENING TEACHING}

BASED ON MULTIMODAL DISCOURSE ANALYSIS THEORY

Multimodal discourse refers to the communication through hearing, vision , smell etc, by the use of various means and symbols such as language, image, sound, action( Zhang De-lu, 2009). In teaching practice, information from different channels can excite more intense response of corresponding functional areas of cerebral cortex than that of single channel ${ }^{[7-8]}$. In appropriate situation, the former is more helpful for individuals to process and memorize information and construct knowledge. However, the complexity of the relationship among modalities and the perishability of information in English Listening also makes it possible that information is distorted and sometimes disappear during the process of output, since students are forced to receive, repeat, process and output information in such a short time.

According to the experiment by Lambert(1981), students exposed to L1 sound and L2 subtitles can get higher scores than those exposed to L2 sound and L2 subtitles, while L2 sound and L1 subtitles can not help students to comprehend listening materials at all. Baltova's study (1999) shows that L1 sound and L2 subtitles is most helpful for students to recall the content of listening materials. Guichon \& Mclornan (2008) proved the effectiveness of multimodality in listening teaching and the assumption that L2 subtitles are more helpful than those of L1. Chinese scholars Long Yufei \& Zhao Pu (2009) studied the effect of interaction between metacognitive strategy and multimodality on English listening.

In this study, we choose Chinese students who are studying English as second language as subjects and conduct an experiment lasting 16 weeks to validate the effects of multimodalities on English listening teaching.

\section{RESEARCH DESIGN}

\section{A. Purpose}

The purpose of the research is to test the effectiveness of multimodality used in English listening teaching, explore factors restricting the optimization of multimodalities and analyze the potentials and demerits of every modality and the combinations of different modalities.

\section{B. Variables and Invariables}

The independent variable unrelated to samples is the way of broadcasting listening materials: audio alone, audio with video, audio with video and L1 subtitles and audio with video and L2 subtitles. The independent variable related to samples is students' competence of utilizing multimodal discourse analysis theory in English listening. The dependent variable is the semantic units in the summary taken by students in the research.

\section{Participants}

One hundred and twenty Chinese-speaking junior students from four classes in the School of Foreign Studies constituted the informant sample. Every class was treated as an independent group from which thirty students were chosen at random as participants. The 
scores of these participants in mid-term and final exam in the second year were processed in one-way analysis of variance and the result showed that the scores of these four groups have no significant difference (mid-term exam: $\mathrm{F}=0.036, \quad \mathrm{P}=0.991>0.05$; final exam: $\mathrm{F}=0.080$, $\mathrm{P}=0.971>0.05$ ) . In the research, group 1 was exposed to audio alone, group 2 to audio with video, group 3 to audio with video and L1subtitles and group 4 to audio with video and L2 subtitles.

\section{Materials, Tools and Procedure}

The research group consisting of four Chinese teachers having taught English listening for more than five years and two foreign teachers from the U.S.A. chose sixteen four-six- minute VOA news report dealing with politics, economy, culture, science, diplomacy etc, and processed them according to the need of the research to ensure the richness of the materials and meet the demands of the subject.

The tools of the research consisted of the statistical analysis of the summary taken by participants and interviews. Every source document was analyzed in terms of semantic units which were deemed central to

\begin{tabular}{|c|c|c|c|c|c|}
\hline Group & $\mathbf{N}$ & $\mathbf{M}$ & SD & $\mathbf{F}$ & $\mathbf{P}$ \\
\hline Group 1 & 30 & 21.40 & 1.793 & \multirow{4}{*}{104.949} & \multirow{4}{*}{0.000} \\
\hline Group 2 & 30 & 22.53 & 1.943 & & \\
\hline Group 3 & 30 & 24.53 & 2.063 & & \\
\hline Group 4 & 30 & 28.67 & .661 & & \\
\hline
\end{tabular}

understand by researchers. They foregrounded main characters, settings and key arguments.

The research was conducted in four multimedia classes. Same tests were conducted simultaneously at every time and followed the same steps strictly: researchers explained the steps of the test to students and then broadcast the material. Students were supposed to take notes in Chinese or English. After the broadcasting, students had five minutes to check their notes individually. During the second broadcasting, students still tried to take notes and check their answers. After the broadcasting, students were asked to finish their summary in English.

\begin{tabular}{lccccc}
\hline Group & N & M & SD & F & P \\
\hline Group 1 & 30 & 20.50 & 1.432 & & \\
\cline { 1 - 4 } Group 2 & 30 & 21.97 & 1.402 & & \\
\cline { 1 - 4 } Group 3 & 30 & 23.47 & 1.224 & & \multirow{2}{*}{0.000} \\
\cline { 1 - 3 } Group 4 & 30 & 27.37 & .809 & & \\
\hline
\end{tabular}

\section{E. Collection and Analysis of Data}

The research consisted of four tests (once a month) and semantic units in the summaries were collected as data and treated by the soft ware SPSS16.0. One-way analysis of variance and Post Hoc Test were used to analyze the data and interview was used when necessary.

\section{RESULTS}

From the sixteen-week research, we find that multimodality can facilitate students' competence of English-listening when used appropriately. The results of the analysis by the use of SPSS16.0 are shown in the following tables.

In the first one-way analysis of variance, the test of homogeneity of variance shows that the data refuses the homogeneity of variance, which means the result of this statistical analysis is invalid. The result, however, is still useful for the research, since it indicates that in the first month, students can not master the complex multimodal discourse analysis theory, let alone utilize it in the practice of English listening. This assumption is also proved by interviews. The result of the second test displays the benefits of multimodality as is shown in table 1:

\begin{tabular}{lccccc}
\multicolumn{5}{c}{ TABLE I } & \multicolumn{4}{c}{ ANVOA OF THE SECOND TEST } \\
\hline Group & N & M & SD & F & P \\
\hline Group 1 & 30 & 18.60 & 2.774 & & \\
\cline { 1 - 3 } Group 2 & 30 & 19.50 & 2.789 & & \\
\cline { 1 - 3 } Group 3 & 30 & 20.055 & 3.559 & & \\
\cline { 1 - 3 } Group 4 & 30 & 24.77 & 2.555 & &
\end{tabular}

As table 1 shows, the impact of different modalities and combinations of them on students' competence has significant difference $(\mathrm{F}=26.055, \mathrm{P}=0.000<0.05)$. Generally speaking, audio with video and L2 subtitles has more potential for facilitating students' competence than audio with video and L1 subtitle, which is more helpful than audio with video in turn. Audio alone has least potential. To test the detailed difference, we use Post Hoc Test to analyze the data and the result is shown in table 2: TABLE II POST HOC TEST

As table 2 shows, the effectiveness of teaching in group 4 is more significant than those in other groups $(\mathrm{P}=0.000<0.05)$. The difference between the effectiveness in group 3 and that in group 1 is evident ( $\mathrm{P}=0.034<0.05)$, while it has no significant difference from that in group $2(\mathrm{P}=0.337>0.05)$. The effectiveness of teaching in group 2 has also no significant difference from that in group $1 \quad(\mathrm{P}=0.239>0.05)$. The result indicates that students can not make full use of video and L1 subtitles to improve the competence of listening. TABLE III ANVOA OF THE THIRD TEST

The data in table 3 shows that the impacts of different combinations of multimodalities have significant difference $(\mathrm{F}=169.758, \mathrm{P}=0.000<0.05)$. To test detailed differences, we use Post Hoc Test to analyze the data and the result is shown is table4: 
TABLE IV POST HOC TEST

\begin{tabular}{cccc}
\hline I & J & MD & P \\
\hline Group 1 & Group 2 & & \\
& Group 3 & -1.467 & 0.000 \\
& Group 4 & -2.967 & 0.000 \\
& & -6.8677 & 0.000 \\
\hline Group 2 & Group 3 & -1.500 & 0.000 \\
& Group 4 & -5.400 & 0.000 \\
\hline Group 3 & Group 4 & -3.900 & 0.000 \\
\hline
\end{tabular}

Table 4 shows the significant differences among these four groups $(\mathrm{P}=0.000<0.05)$, and the result of the fourth test also proves this conclusion as is shown in table 5 :

TABLE $V$ ANVOA OF THE FOURTH TEST

The result of Post Hoc Test is shown in table6:

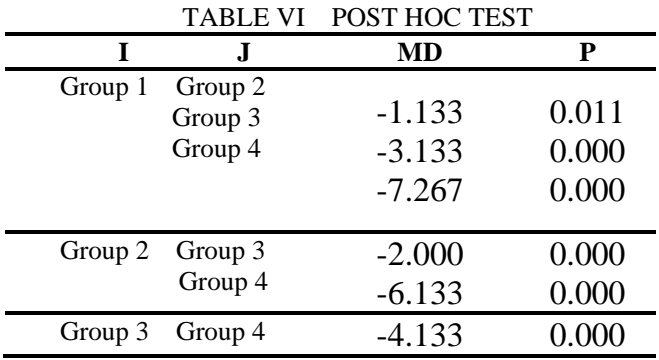

\section{DISCUSSION}

\section{A. Benefits and Limitations of Multimodalities}

Every individual modality has merits and demerits in expressing meanings and has the potential of improving students competence of English listening. However, the utilization of multimodality depends on students' cognitive capacity.

Audio is the main tool in traditional English listening class and has its advantage in training students' listening skills. However, in this research, it is proved that the effectiveness of audio alone is not as significant as that of multimodality.

Only when visual information is complementary to audial information, can it promote students' competence of listening. For example, the news in the first test(all examples in this paper are from VOA news "History of American West”) mentions that President Roosevelt lived in North Dakota. Students ignoring this semantic unit in group 2 are more than those in group 1 . According to the interview, this information was told by an employee in the gallery and the visual information is not complementary to the audial information.

Subtitles appear to be a determining factor for understanding some information, especially some ambiguous semantic units. For example, although all students in these four groups understand the meaning of the word "overall”, few in group 1 and group 2 have taken down it in their summaries.

When listening to the same material, students exposed to L2subtitles can have better understanding of the information than those exposed to L1subtitles. It is clear that mistakes tend to occur when the information is translated back into students’ L1.

\section{B. Factors Restricting Optimal Combination of multimodality}

Firstly, the genre of the material affects students' understanding of the information. According to the research, science is most difficult to understand, followed by politics, economy, military affairs, culture and literature. Secondly, teachers are no more simple tape-players and multimedia operators. Instead, they are the designers of multimodality, regulators, supervisors as

\begin{tabular}{|c|c|c|c|c|c|}
\hline Group & $\mathbf{N}$ & $\mathbf{M}$ & SD & $\mathbf{F}$ & $\mathbf{P}$ \\
\hline Group 1 & 30 & 21.40 & 1.793 & \multirow{4}{*}{104.949} & \multirow{4}{*}{0.000} \\
\hline Group 2 & 30 & 22.53 & 1.943 & & \\
\hline Group 3 & 30 & 24.53 & 2.063 & & \\
\hline Group 4 & 30 & 28.67 & .661 & & \\
\hline
\end{tabular}

well as evaluators. Thirdly, Students' purpose of study, competence, interest and motivation determine wether they can master multimodal discourse analysis theory and skills of utilizing multimodality.

\section{CONCLUSION}

This research shows that reasonable utilization of the combination of multimodalities can improve learners' listening competence; the choice of the combination of multimodalities depends on the context of situation; What's more, visual information can give play to positive function providing that it is complementary to auditory information; In addition, L2 subtitling is more beneficial than L1 because it causes less lexical interference.

\section{ACKNOWLEDGMENT}

This study is supported by the program of master's scientific research at Suzhou University under grand no. 2009YSS24 and the program of young talents in universities in Anhui Province under grand no. 2010SQRW146.

\section{REFERENCES}

[1] Baltova, I. Multisensory Language Teaching in a Multidimensional Curriculum: The Use of Authentic Bimodal Video in Core French. Canadian Modern Language Review, 1999, vol. 1, pp. 32-48.

[2] Guichon, N. \& McLornan, S. The effects of multimodality on L2 learners: Implications for CALL resource design. System, 2008, vol. 1 , pp. 85-93.

[3] http://video.sina.com.cn/v/b/31157247-11541701 73.html

[4] Jonassen, D H, Land, S M. Theoretical Foundations of Learning Environment. New York: Lawrence Erlbaum association, 2000.

[5] Lambert, et.al. Choosing the Languages of Subtitles and Spoken Dialogues for Media Presentations: Implications for Second Language Education. Applied Psycholinguistics, 1981, vol. 2, pp. 133-144.

[6] Chen Qi \& Zhang Jianwei. A Model of Integrate Learning in the Information Era. Peking University Education Review, 2003, vol. 3, pp. 90-96.

[7] Long Yufei \& Zhao Pu. The Interaction Study between Multimodality and Meta-cognitive Strategy in College English Listening Comprehension Teaching. Computer-assisted Foreign Language 
Education, 2009, vol. 4, pp.58-62.

[8] Zhang De-lu. On a Synthetic Theoretical Framework for Multimodal

Discourse Analysis. Foreign Language in China, 2009, vol. 1, pp. 24-30. 